\title{
Impossibility results for infinite-electorate abstract aggregation rules*
}

\author{
Frederik Herzberg ${ }^{\dagger \ddagger}$ and Daniel Eckert ${ }^{\S}$ \\ Copyright notice: The final publication, in the Journal of Philosophical Logic \\ 41 (2012), no. 1, pp. 273-286, is available at link.springer.com, \\ doi:10.1007/s10992-011-9203-5
}

\begin{abstract}
Following Lauwers and Van Liedekerke (1995), this paper explores in a model-theoretic framework the relation between Arrovian aggregation rules and ultraproducts, in order to investigate a source of impossibility results for the case of an infinite number of individuals and an aggregation rule based on a free ultrafilter of decisive coalitions.
\end{abstract}

Key words: Arrow-type preference aggregation; judgment aggregation; model theory; first-order predicate logic; filter; ultrafilter; reduced product; ultraproduct; existential quantifier

2010 Mathematics Subject Classification: 91B14; 03C20; 03C98

Journal of Economic Literature classification: D71

*This work was partially supported by a German Research Foundation (DFG) grant. We are very grateful to two anonymous referees who helped us tremendously in improving this paper.

${ }^{\dagger}$ Department of Mathematics, Princeton University, Fine Hall - Washington Road, Princeton, New Jersey 08544-1000, United States of America. fherzberg@math.princeton.edu

${ }^{\ddagger}$ Institut für Mathematische Wirtschaftsforschung, Universität Bielefeld, Universitätsstraße 25, D-33615 Bielefeld, Germany. fherzberg@uni-bielefeld.de

$\S$ Institut für Finanzwissenschaft, Karl-Franzens-Universität Graz, Universitätsstraße 15/E4, A-8010 Graz, Austria. daniel.eckert@uni-graz.at 


\section{Introduction}

In the last decades, the literature on social choice theory has seen important generalisations of the classical Arrovian problem of preference aggregation, starting with isolated contributions on abstract and algebraic aggregation theory by Wilson 22] resp. by Rubinstein and Fishburn 20] and culminating in the new field of judgment aggregation (for a survey see List and Puppe [18]). An essential feature of these generalisations is the extension of the problem of aggregation from the aggregation of preferences to the aggregation of arbitrary information. It thus seems natural to exploit the potential of model theory, which, broadly speaking, studies the relation between abstract structures and statements about them (for an introduction to model theory, see Bell and Slomson 2]) and to analyse the problem of judgment aggregation as the problem of aggregating the models that satisfy the corresponding sentences (judgments).

This approach is justified mathematically by the fact that one of the major tools of model theory, namely the ultraproduct construction, corresponds to the construction of an aggregation rule satisfying axioms in the spirit of the conditions in Arrow's famous impossibility theorem [1. At the heart of this coincidence is a correspondence between Arrovian aggregation rules and ultrafilters of decisive coalitions, which for the case of preference aggregation dates back to a celebrated paper by Kirman-Sondermann 15 (with a precursor in Fishburn [7] and Hansson [10, Postscript: 1971]) and has recently been generalised to a model-theoretic framework (see Herzberg and Eckert [12, who built on Lauwers' and Van Liedekerke's seminal work [17]).

For the case of a finite set of individuals, the Kirman-Sondermann [15] correspondence (and its model-theoretic generalisation) between aggregation rules and ultrafilters immediately implies a dictatorship result, as ultrafilters on finite sets are necessarily principal, whence the ultrafilter on a finite set of individuals always is the set of all supersets of a singleton - the dictator.

Whilst this dictatorship result does not carry over to the case of an infinite electorate (where free ultrafilters exist), it is well known since Kirman's and Sondermann's [15] identification of "invisible dictators" that free ultrafilters only guarantee a limited amount of anonymity (as was also shown by Lauwers and Van Liedekerke [17] in their model-theoretic framework and by Dietrich and Mongin [5] in the framework of judgment aggregation). On the other hand, the selection of one of the numerous free ultrafilters entails some striking inherent arbitrariness, as was also pointed out by Lauwers and Van Liedekerke [17.1

Perhaps even more interestingly, the latter also have suggested another source of impossibility results, viz. the existence of non-universal formulae in the electorate's agenda (e.g. formulae which describe the existence of a best alternative or continuity of preferences). In this case the application of the Paretian criterion of the preservation of unanimously accepted sentences leads to a dictatorship even in the case of an infinite number of individuals.

In this paper, we explore this suggestion by Lauwers and Van Liedekerke [17] further: In a model-theoretic framework (which allows for the analysis of preference aggregation as well as of propositional and modal propositional

\footnotetext{
${ }^{1}$ As one of the referees remarked, free ultrafilters are non-constructible objects (even though their existence is not equivalent to the Axiom of Choice, cf. Halpern and Levy [9]) and thus the existence of aggregation rules with certain desirable properties based on free ultrafilters (as in Fishburn [7]) should be considered a rather weak "possibility result" anyway.
} 
judgment aggregation), we prove a theorem about the general impossibility of non-dictatorial Arrovian aggregators that preserve certain unanimously accepted non-universal formulae.

The next section summarizes the results of this paper, while the modeltheoretic framework and the results of this article are presented in Section 3 and Section 4 A brief discussion of these results and further applications concludes the paper.

\section{A source of impossibility results for infinite populations}

In its most general formulation, the problem of aggregation consists in finding a rule which assigns to each profile of individual charateristics a representative collective characteristic of the same type (e.g. to a profile of individual preferences, a collective preference relation is associated).

Thus, an abstract aggregation rule can be defined by a mapping $f: \Omega^{I} \rightarrow$ $\Omega$ where $\Omega$ is a set of possible characteristics and $I$ a set of indivuals. In traditional Arrovian social choice theory, $\Omega$ is the set of preference relations on some alternatives, while in the new literature on judgment aggregation it is the set of all judgment sets from a given agenda of propositions.

This paper works within a unified approach to aggregation theory based on the model theory of first-order predicate logic. Just as propositional logic, modal logic, and predicate logic with a single predicate are all special cases of first-order predicate logic, one can view judgment aggregation, modal judgment aggregation and preference aggregation as special cases of the general problem of aggregating sentences of first-order predicate logic. Thus we formulate the aggregation problem as the problem of aggregating the models that correspond to these sentences ${ }^{2}$

The advantage of such a unified approach to aggregation theory is twofold:

(i) At a conceptual level, this approach provides a natural common language in which results and methods from both preference and judgment aggregation can be phrased, thus facilitating the translation of results and methods from one area to the other ${ }^{3}$

(ii) From a technical vantage point, the model-theoretic approach best allows to establish the correspondence between Arrovian aggregation rules and ultrafilters of decisive coalitions which underlies the dictatorship results for the aggregation both of preferences and judgments.

Since the path-breaking work of Kirman and Sondermann [15] and Hansson [10] it has been well-known that the properties of Arrovian aggregation rules (essentially combinations of a Pareto and an independence condition of various strengths) together with assumptions on the logical structure of the aggregation problem (transitivity of the preference relations in the case of preference aggregation, logical richness of the agenda in the case of judgment aggregation)

\footnotetext{
${ }^{2} \mathrm{~A}$ model is a relational structure consisting of a number of relations on a given domain which gives meaning to the sentences of a formal language. If a given sentence holds true under this interpretation, the model is said to satisfy that sentence.

${ }^{3}$ From a historical point of view, there is evidence for the influence of the founding father of model theory, Tarski on Arrow's foundation of social choice theory [21].
} 
induce a particular structure of the set of decisive coalitions $4^{4}$. Ultimately, this decisiveness structure amounts to an oligarchy, i.e. to the existence of a subset of individuals which must be contained in every decisive coalition, or even to a dictatorship, when this set is a singleton.

In the model-theoretic approach to aggregation theory, one can mimick the arguments for the special cases of preference aggregation and judgment aggregation (cf. Dietrich and Mongin [5] as well as Eckert and Klamler [16]) to prove that Arrovian aggregator axioms together with assumptions on the logical structure of the aggregation problem imply that the set of decisive coalitions forms a filter $5^{5}$ or, under stronger assumptions, even an ultrafilter ${ }^{6}$. Conversely, given any filter or ultrafilter $\mathcal{D}$, one can define an Arrovian aggregator whose set of decisive coalitions is precisely $\mathcal{D}$. Let us call such an aggregator a $\mathcal{D}$ aggregator or $\mathcal{D}$-(ultra)filter rule.

As a consequence of this correspondence, Arrovian aggregators are nothing else but filter rules or even ultrafilter rules.

For finite populations this readily implies that the aggregation rule must be oligarchic or even dictatorial 7

Now, if the domain of an aggregation rule is a set of models, an Arrovian aggregator corresponds to a particular model-theoretic construction, namely the reduction of a profile of models over a filter $\mathcal{D}$. Such a reduced product satisfies precisely those sentences which are accepted in those coalitions which are members of $\mathcal{D}$. In particular, by a fundamental theorem in model theory due to Łoś, a sentence holds true in an ultraproduct (i.e. in the reduced product of a profile of models over an ultrafilter) if and only if the coalition of individuals in the models in which it holds true is member of the corresponding ultrafilter. Thus, a $\mathcal{D}$-filter rule is just the restriction of the reduced product (with respect to $\mathcal{D}$ ) to the original domain of the individual models (remember that that collective outcome of an aggregation rule is assumed to be of the same type as the individual characteristics and hence to have the same domain), and a $\mathcal{D}$ ultrafilter rule is the restriction of the ultraproduct (with respect to the ultrafilter D) to the original domain (cf. Lauwers and Van Liedekerke [17]).

Now, restrictions of reduced products or ultraproducts (i.e. Arrovian aggregators) do not in general preserve formulae in which existential quantifiers occur, except when the underlying filter or ultrafilter (i.e. the set of decisive coalitions) is merely the set of supersets of some coalition (i.e. an oligarchy or dictatorship) - even for infinite electorates. In the setting of preference aggregation, this means that an Arrovian aggregator which is supposed to preserve either continuity or the existence of a maximal (or minimal) alternative must be a dictatorship (cf. Lauwers and Van Liedekerke [17] and earlier work by Campbell [3]), even if the population is infinite. The present paper shows that the same phenomenon occurs in more general aggregation problems: For

\footnotetext{
${ }^{4} \mathrm{~A}$ subset of individuals is decisive (with respect to a given characteristic) if the collective outcome always coincides with the respective characteristic of the individuals in this coalition, e.g. if the collective ranking of a given pair of alternatives always coincides with the individual ranking of this pair by the members of the coalition.

${ }^{5} \mathrm{~A}$ filter (on the population set) is a system of non-empty subsets of the population (coalitions) which is closed under both supersets and intersections.

${ }^{6}$ An ultrafilter (on the population set) is a filter $\mathcal{D}$ with the additional property that for every coalition $C$ in the population, either $C$ or its complement is an element of $\mathcal{D}$.

${ }^{7}$ Indeed, for every filter $\mathcal{D}$ on a finite set $I$, there exists a set $D$ such that for every $C \subseteq I$, one has $C \in \mathcal{D}$ if and only if $C \supseteq D$, and if $\mathcal{D}$ is an ultrafilter, then this set $D$ is a singleton.
} 
example, even if the electorate is infinite, any judgment aggregator which satisfies Arrovian axioms and, in addition, preserves the existence of an implication-maximal agenda item must be an oligarchy or a dictatorship. Similarly, every modal propositional aggregator which satisfies Arrovian axioms and preserves a non-trivial existential statement about possible worlds must be an oligarchy or a dictatorship.

\section{A model-theoretic framework for abstract aggregation theory}

In this paper, we shall work within a model-theoretic framework, in which Lauwers' and Van Liedekerke's [17] model-theoretic approach to preference aggregation (with a recent addendum by Herzberg et al. 13]) was carried over to more abstract aggregation problems. 8

Let $A$ be an arbitrary set ${ }^{9}$ Let $\mathcal{L}$ be a language consisting of at most countably many predicate symbols $\dot{P}_{n}, n \in \mathbf{N}$.

The set of constant symbols will simply be the set $A$; however, within formulae of the object language, we shall normally write $\dot{a}$ instead of $a$ (for any $a \in A$ ). The arity of $\dot{P}_{n}$ will be denoted $\delta(n)$, for all $n \in \mathbf{N}$. (Following common practice in mathematical logic, we use dots to distinguish symbols of the object language, i.e. the formal language $\mathcal{L}$ under consideration, from the symbols used in the meta language, i.e. the language of our present mathematical discourse.)

Let $T$ be a consistent set of universal sentences in $\mathcal{L}{ }^{10}$ (In the case of preference aggregation, for example, $A$ would be the set of alternatives, there would be just one binary predicate symbol, and $T$ would consist of the weak order axioms.)

The relational structure $\mathfrak{B}=\left\langle B,\left\{P_{n}: n \in \mathbf{N}\right\}\right\rangle$ with $A \subseteq B$ is called a realisation of $\mathcal{L}$ with domain $B$ or an $\mathcal{L}$-structure if and only if the arities of the relations $P_{n}$ correspond to the arities of the predicate symbols $\dot{P}_{n}$, that is, if $P_{n} \subseteq B^{\delta(n)}$ for each $n$. In this paper, we only allow for relational structures which interpret every constant symbol $a \in A$ canonically as $a$.

An $\mathcal{L}$-structure $\mathfrak{B}$ is a model of the theory $T$ if $\mathfrak{B} \models \varphi$ for all $\varphi \in T$, i.e. if all sentences of the theory hold true in $\mathfrak{B}$ (with the usual Tarskian definition of truth ${ }^{11}$.

Let $\mathfrak{B}=\left\langle B,\left\{P_{n}: n \in \mathbf{N}\right\}\right\rangle$ be an $\mathcal{L}$-structure with domain $B$. (Note that this entails $A \subseteq B$ by our convention.) According to standard model-theoretic terminology (cf. e.g. Bell and Slomson [2, p. 73]), the restriction of $\mathfrak{B}$ to $A$ is the $\mathcal{L}$-structure $\left\langle A,\left\{P_{n} \cap A^{\delta(n)}: n \in \mathbf{N}\right\}\right\rangle$ and will be denoted by $\operatorname{res}_{A} \mathfrak{B}$. (In other words, the restriction of $\mathfrak{B}$ to $A$ is the $\mathcal{L}$-structure that is obtained by restricting the interpretations of the relation symbol to the domain $B \subseteq A$.)

\footnotetext{
${ }^{8}$ For a derivation of the Kirman Sondermann correspondence in this framework see (Herzberg and Eckert [12]).

${ }^{9}$ The set $A$ may be arbitrary for our conceptual framework, but the main results of this paper are non-trivial only for infinite $A$, as they concern existential quantification over $A$.

${ }^{10} \mathrm{~A}$ sentence is universal if it (in its prenex normal form) has the form $\left(\dot{\forall} \dot{v}_{k_{1}}\right) \cdots\left(\dot{\forall} \dot{v}_{k_{m}}\right) \psi$ for some formula $\psi$ that does not contain any quantifiers.

${ }^{11}$ For instance, if $\mathfrak{B}=\left\langle B,\left\{P_{n}: n \in \mathbf{N}\right\}\right\rangle$ is an $\mathcal{L}$-structure, then for all $a_{1}, \ldots, a_{\delta(n)} \in A$, one has

$$
\mathfrak{B} \models \dot{P}_{n}\left(a_{1}, \ldots, a_{\delta(n)}\right) \Leftrightarrow\left\langle a_{1}, \ldots, a_{\delta(n)}\right\rangle \in P_{n} .
$$
}


Suppose now that $\mathfrak{B}=\left\langle B,\left\{P_{n}: n \in \mathbf{N}\right\}\right\rangle$ is a relational structure with $P_{n} \subseteq B^{\delta(n)}$ for each $n$ and such that there exists an injective map $i$ : $A \rightarrow B$. Then, the restriction of $\mathfrak{B}$ to $A$ under $i$ is the $\mathcal{L}$-structure $\left\langle A,\left\{i^{-1}\left[P_{n} \cap i[A]^{\delta(n)}\right]: n \in \mathbf{N}\right\}\right\rangle$ and will be denoted by $\operatorname{res}_{i, A} \mathfrak{B}$. If $B$ is the reduced product of $A$ with respect to some filter $D$ and $i: A \rightarrow B, \quad a \mapsto[(a)]_{D}$, is the canonical embedding, then we will drop the subscript $i$ and simply write $\operatorname{res}_{A} \mathfrak{B}$ instead of $\operatorname{res}_{i, A} \mathfrak{B}$.

Let $\Omega$ be the collection of models of $T$ with domain $A$.

Let $I$ be a (finite or infinite) set. Elements of $I$ will be called individuals, elements of $\Omega^{I}$ will be called profiles.

An aggregator is a map with domain $\subseteq \Omega^{I}$ and range $\subseteq \Omega$ (so that individual characteristics and collective characteristics are of the same type) ${ }^{12}$

As Herzberg and Eckert [12] have pointed out, this framework is sufficiently general to cover the cases of preference aggregation, propositional judgment aggregation, and modal judgment aggregation:

- For the case of preference aggregation, the centrality of binary relations makes it particularly natural to express preferences by a binary predicate in first order logic (cf. Rubinstein [19], Lauwers and Van Liedekerke [17]). A more elaborate formalisation and complete axiomatisation of Arrow's theorem in first order logic was recently given by Grandi and Endriss [8].

- For propositional judgment aggregation à la Dietrich and List 4, one lets $\mathcal{L}$ have a single unary predicate $\dot{B}$, modelling a belief operator. The set $A$ will be the agenda. The interpretation of $\dot{B} a$ is " $a$ is accepted". (Thus, the interpretation of $\mathfrak{A}_{i} \models \dot{B} a$ is "under profile $\underline{\mathfrak{A}}$, individual $i$ accepts $a$ ", and the interpretation of $f_{\mathcal{D}}(\underline{\mathfrak{A}}) \models \dot{B} a$ is "under profile $\mathfrak{A}, a$ is collectively accepted".) $T$ can be any universal theory in that language.

- For modal judgment aggregation, one simply uses the reduction of modal logic to first-order predicate logic, where the elements of the domain correspond to possible states of the world. Thus, the set $A$ will be the set of states of the world. Let there be in $\mathcal{L}$ a predicate $M_{p}$ for each element $p$ of the agenda, modelling a modal belief operator with world argument and proposition index. Let there also be a binary predicate $\dot{R}$ in $\mathcal{L}$, denoting the accessibility relation. The interpretation of $\dot{R}(a, b)$ will thus be " $b$ is accessible from world $a$ ". The interpretation of $\dot{M}_{p} a$ will be "proposition $p$ is accepted in world $a$ ". (The interpretation of $\mathfrak{A}_{i} \models \dot{M}_{p} a$ is thus "under profile $\mathfrak{A}$, individual $i$ accepts $p$ in world $a$ ", and the interpretation of $f_{\mathcal{D}}(\underline{\mathfrak{A}}) \models \dot{M}_{p} a$ is "under profile $\underline{\mathfrak{A}}, p$ is collectively accepted in world $a$ ".) The modal operator $\square$ will then not be an operator in the strict sense any longer, but it can be defined as a family of sentences, indexed by $p$ :

$$
\square_{p} \dot{v}_{0}: \equiv \dot{(} \dot{\forall} \dot{v}_{1} \ddot{)} \dot{R}\left(\dot{v}_{0}, \dot{v}_{1}\right) \rightarrow \dot{M}_{p} \dot{v}_{1} \dot{)}
$$

The interpretation of $\square_{p} a$ is " $p$ is accepted in all worlds which are accessible from world $a$ ", or just " $p$ is necessarily accepted in world $a$ ". $T$ can be any universal theory in that language, which includes the axioms of the modal logical system employed (such as K, S4, S5, etc.).

\footnotetext{
${ }^{12}$ In fact, all aggregators considered in this paper are restricted reduced products (ultraproducts), whence their domain equals $\Omega^{I}$ and their range equals $\Omega$.
} 
Generalising the Kirman-Sondermann [15] correspondence between Arrovian social welfare functions and ultrafilters of decisive coalitions 13 on the set of individuals, Herzberg and Eckert [12 — following a seminal paper by Lauwers and Van Liedekerke [17] as well as recent work by Dietrich and Mongin [5, 6] - have shown that given certain axioms, inspired by Arrow [1, on $f$ and some assumptions on the logical richness of $\mathcal{L}$, every aggregator is in fact given by a restricted reduced product construction with respect to the filter of decisive coalitions. Under additional assumptions, this filter will be an ultrafilter. Conversely, any filter on the population set can be used to define an Arrovian aggregator. Given this one-to-one correspondence between filters and aggregators, we may simply fix an arbitrary filter $\mathcal{D}$ on $I$ and define an aggregator $f_{\mathcal{D}}$, the $\mathcal{D}$-aggregator as the map which assigns to each profile $\mathfrak{A} \in \Omega^{I}$ the restriction of the reduced product ${ }^{14} \prod_{i \in I} \mathfrak{A}_{i} / \mathcal{D}$ to the original domain $A$ :

$$
\forall \underline{\mathfrak{A}} \in \Omega^{I} \quad f_{\mathcal{D}}(\underline{\mathfrak{A}})=\operatorname{res}_{A} \prod_{i \in I} \mathfrak{A}_{i} / \mathcal{D} \text {. }
$$

Observe that the restriction to $A$ is essential since it is a necessary condition (for $f_{\mathcal{D}}$ to be an aggregator) that the aggregate model $f_{\mathcal{D}}(\underline{\mathfrak{A}})$ belongs to $\Omega$ and thus must have $A$ as its domain. Moreover, if $\mathcal{D}$ is an ultrafilter, then, by application of Łos's theorem, for every $\mathcal{L}$-sentence $\psi$,

$$
\prod_{i \in I} \mathfrak{A}_{i} / \mathcal{D} \mid=\psi \Leftrightarrow\left\{i \in I: \mathfrak{A}_{i} \models \psi\right\} \in \mathcal{D},
$$

which guarantees that $\prod_{i \in I} \mathfrak{A}_{i} / \mathcal{D} \models T$ and hence $f_{\mathcal{D}}(\underline{\mathfrak{A}})=\operatorname{res}_{A} \prod_{i \in I} \mathfrak{A}_{i} / \mathcal{D} \models T$ since $T$ consists only of universal sentences. Therefore, if $f_{\mathcal{D}}$ is given as the restriction of an ultraproduct to $A$, then $f_{\mathcal{D}}(\underline{\mathfrak{A}}) \in \Omega$ for all profiles $\underline{\mathfrak{A}} \in \Omega^{I}$.

\footnotetext{
${ }^{13}$ In our framework, a subset $S \subseteq I$ of individuals is a decisive coalition if there exists some $\mathcal{L}$-sentence $\psi$ such that both $f_{\mathcal{D}}(\underline{\mathfrak{A}}) \models \psi$ and

$$
S=\left\{i \in I: \mathfrak{A}_{i} \models \psi\right\} .
$$
}

If $f$ satisfies some Arrovian [1] (responsiveness) axioms, one can show that the set of decisive coalitions forms a filter, i.e. a collection of non-empty subsets of $I$ which is closed under finite intersections and supersets, and under stronger assumptions even an ultrafilter, i.e. a maximal filter (cf. Herzberg and Eckert [12, generalising similar findings by Kirman and Sondermann [15], Lauwers and Van Liedekerke [17, Dietrich and Mongin [5]). Note that under these conditions on $f$, it even makes no difference if one replaces the " $S=$ " in the above definition of a decisive coalition by " $S \subseteq$ " and "there exists some $\psi$ " by "for all $\psi$ ".

From the ultrafilter property of the set of decisive coalitions, one can immediately deduce Arrow's theorem by noting that every ultrafilter on a finite set is principal, i.e. its intersection equals a singleton (the element of this singleton being the dictator if the ultrafilter is a set of decisive coalitions). Non-principal ultrafilters are called free.

${ }^{14}$ The reduced product $\prod_{i \in I} \mathfrak{A}_{i} / \mathcal{D}$ is the set of equivalence classes of sequences of alternatives with respect to the equivalence relation $\sim_{\mathcal{D}}$, defined as

$$
\underline{a} \sim{ }_{\mathcal{D}} \underline{a}^{\prime} \Leftrightarrow\left\{i \in I: a_{i}=a_{i}^{\prime}\right\} \in \mathcal{D}
$$

for all $\underline{a}, \underline{a}^{\prime} \in A^{I}$. Similarly, one can define interpretations $P_{n}^{\Pi}$ of the relation symbols $\dot{P}_{n}$, $n \in \mathbf{N}:$ For all $\underline{a}, \underline{a}^{\prime} \in A^{I}$

$$
\left\langle[\underline{a}]_{\mathcal{D}},\left[\underline{a}^{\prime}\right]_{\mathcal{D}}\right\rangle \in P_{n}^{\Pi}: \Leftrightarrow\left\{i \in I:\left\langle a_{i}, a_{i}^{\prime}\right\rangle \in P_{n}^{\mathfrak{A}_{i}}\right\} \in \mathcal{D}
$$

Since $\mathcal{D}$ is a filter, $\sim_{\mathcal{D}}$ is an equivalence relation, which also implies that $P_{n}^{\Pi}$ is welldefined. Hence, there is an interpretation of $\dot{P}_{n}$ in the reduced product $\prod_{i \in I} \mathfrak{A}_{i} / \mathcal{D}$, whence $\left\langle\prod_{i \in I} \mathfrak{A}_{i} / \mathcal{D},\left\{P_{n}^{\Pi}: n \in \mathbf{N}\right\}\right\rangle$ is an $\mathcal{L}$-structure. The set $A$ can be canonically embedded into $\prod_{i \in I} \mathfrak{A}_{i} / \mathcal{D}$ through $a \mapsto\left[(a)_{i \in I}\right]_{\sim_{\mathcal{D}}}$. Therefore, one can restrict the $\mathcal{L}$-structure of the reduced product to $A$ and obtain an $\mathcal{L}$-structure with domain $A$. 


\section{Impossibility theorems for infinite populations}

In the case of a finite number of individuals dictatorship results immediately follow from the principality of any ultrafilter on a finite set. For the case of an infinite set of individuals there exist free ultrafilters and therefore Arrow's impossibility theorem does not apply (as was already shown by Fishburn [7]).

However, the very construction of an ultraproduct bears another source of impossibility results as remarked by Lauwers and Van Liedekerke [17]: Ultraproducts with respect to free ultrafilters have a strictly larger domain than the factor structures, and thus, witnesses to certain existential statements in the ultraproduct do not need to belong to the domain of the factor structures (cf. Hodges 14 for a more comprehensive discussion of the role of ultraproducts for the construction of extensions of given structures). Therefore, if an aggregator is the restriction (to the factor domain) of an ultraproduct ${ }^{15}$ and, by application of a Pareto condition, is required to preserve some unanimously held non-universal statement (for example: existence of a best alternative; continuity; etc.), it must be the restriction of an ultraproduct with respect to a principal ultrafilter and will thus be dictatorial.

Indeed, Lauwers and Van Liedekerke [17] have remarked that in the setting of preference aggregation, the preservation of non-universal formulae generically leads to dictatorial impossiblity results (e.g. Campbell's theorem on the translation of the Arrovian dictatorship result to infinite populations when preferences are assumed to be continuous [3]). The same phenomenon can be observed in the more general setting of first-order predicate aggregation theory.

In order to illustrate this, let us consider the simplest case, viz. preservation of formulae with exactly one, existential quantifier in a restricted ultraproduct construction. Suppose hence $\psi=\dot{(} \dot{\exists} \dot{v}) \phi(\dot{v})$ for some $\mathcal{L}$-formula $\phi(\dot{v})$ with one free variable, assume $I$ is infinite, let $\mathcal{D}$ be an ultrafilter on $I$, and consider a profile $\underline{\mathfrak{A}}=\left\langle\mathfrak{A}_{i}\right\rangle_{i \in I}$ of models of $T$, all with the same domain $A$. Suppose that whilst $\dot{(} \dot{\exists} \dot{v} \dot{\phi} \phi(\dot{v})$ holds true in all models $\mathfrak{A}_{i}$, there does not exist a $\mathcal{D}$-almost uniform witness (i.e. there exists no $a \in A$ such that the set of $i \in I$ with $\mathfrak{A}_{i}=\phi(a)$ would belong to $\left.\mathcal{D}\right)$. Then, Eoś's theorem teaches that $\phi(a)$ fails in $\prod_{i \in I} \mathfrak{A}_{i} / \mathcal{D}$ for all $a \in A$, and therefore $\psi$ cannot hold true in the restriction of $\prod_{i \in I} \mathfrak{A}_{i} / \mathcal{D}$ to $A$.

This phenomenon can be used as a source of more general impossibility theorems in abstract aggregation theory: In this paper, we will prove an impossibility theorem for aggregators which preserve some non-universal formula.

Consider an arbitrary $\mathcal{L}$-sentence which is not universal. In its prenex normal form it can be written as $\left.\left.\psi \equiv \dot{(\forall} \dot{x}_{1} \dot{)} \ldots \dot{(} \dot{\forall} \dot{x}_{m}\right) \dot{(} \dot{\exists} \dot{y}\right) \phi\left(\dot{x}_{1}, \ldots, \dot{x}_{m} ; \dot{y}\right)$, wherein $m$ is a nonnegative integer and $\phi\left(\dot{x}_{1}, \ldots, \dot{x}_{m} ; \dot{y}\right)$ is an $\mathcal{L}$-formula with $m+1$ free variables. For the rest of this paper, $\psi$ and $\phi$ are fixed in this manner.

We say that a profile $\mathfrak{A} \in \Omega^{I}$ has finite witness multiplicity with respect to $\psi$ if and only if $\mathfrak{A}_{i} \models \psi$ for all $i \in I$, but for all $a_{1}, \ldots, a_{m}, a^{\prime} \in A$, the coalition $\left\{i \in I: \mathfrak{A}_{i}=\phi\left(a_{1}, \ldots, a_{m} ; a^{\prime}\right)\right\}$ is finite.

An aggregator $f_{\mathcal{D}}$ is said to preserve an $\mathcal{L}$-sentence $\psi$ if and only if for all $\underline{\mathfrak{A}} \in \operatorname{dom}(f)$, one has $f_{\mathcal{D}}(\underline{\mathfrak{A}}) \models \psi$ whenever $\mathfrak{A}_{i} \models \psi$ for all $i \in I$.

\footnotetext{
${ }^{15}$ For instance, Arrovian preference aggregators always map every profile to the restriction - to the set of alternatives - of its ultraproduct with respect to the ultrafilter of decisive coalitions, cf. Lauwers and Van Liedekerke [17.
} 
We say that $\phi$ is free of negation, disjunction and universal quantification if and only if its non-abbreviated form does not contain the symbols $\dot{\neg}, \dot{V}$ nor $\dot{\forall}$, in other words, if the only logical symbols appearing in it are $\dot{\wedge}$ and $\dot{\exists}$. Henceforth we assume $\phi$ to possess this property $!^{0}$

With this terminology, we have the following impossibility theorem:

Theorem 4.1. Let $\mathcal{D}$ be a filter and suppose $f_{\mathcal{D}}$ preserves $\psi$, and assume that there exists some $\underline{\mathfrak{A}} \in \Omega^{I}$ with finite witness multiplicity with respect to $\psi$.

1. If $\mathcal{D}$ is an ultrafilter, then it is even principal (whence $f_{\mathcal{D}}$ is a dictatorship).

2. $\mathcal{D}$ contains a finite coalition (whence $f$ is an oligarchy).

Proof of Theorem 4.1. 1. Since $f_{\mathcal{D}}(\underline{\mathfrak{A}})$ is just the $A$-restriction of the ultraproduct of $\mathfrak{A}$ with respect to $\mathcal{D}$, Łoś's theorem readily yields the equivalence

$$
\begin{aligned}
& f_{\mathcal{D}}(\underline{\mathfrak{A}}) \models \phi\left(a_{1}, \ldots, a_{m} ; a^{\prime}\right) \\
& \Leftrightarrow \quad\left\{i \in I: \mathfrak{A}_{i} \models \phi\left(a_{1}, \ldots, a_{m} ; a^{\prime}\right)\right\} \in \mathcal{D}
\end{aligned}
$$

for all $a_{1}, \ldots, a_{m}, a^{\prime} \in A$. Since $\underline{\mathfrak{A}}$ is assumed to have finite witness multiplicity with respect to $\psi$, we know that $\left\{i \in I: \mathfrak{A}_{i} \models \phi\left(a_{1}, \ldots, a_{m} ; a^{\prime}\right)\right\}$ is finite for all $a_{1}, \ldots, a_{m}, a^{\prime} \in A$, and that $\mathfrak{A}_{i} \models \psi$ for all $i \in I$, whence $f_{\mathcal{D}}(\underline{\mathfrak{A}}) \models \psi$ as $f$ preserves $\psi$. Therefore, for all $a_{1}, \ldots, a_{m} \in A$ there is some $a^{\prime} \in A$ such that $f_{\mathcal{D}}(\underline{\mathfrak{A}}) \models \phi\left(a_{1}, \ldots, a_{m} ; a^{\prime}\right)$, hence

$$
\left\{i \in I: \mathfrak{A}_{i} \models \phi\left(a_{1}, \ldots, a_{m} ; a^{\prime}\right)\right\} \in \mathcal{D}
$$

by equivalence (1), although

$$
C_{\vec{a}, a^{\prime}}:=\left\{i \in I: \mathfrak{A}_{i} \models\left(a_{1}, \ldots, a_{m} ; a^{\prime}\right)\right\}
$$

is finite. Thus, the ultrafilter $\mathcal{D}$ contains a finite subset of $I$, viz. $C_{\vec{a}, a^{\prime}}$. But then, $\mathcal{D}$ must already be principal, namely $\mathcal{D}=\{C \subseteq I: i \in C\}$ for some individual $i \in C_{\vec{a}, a^{\prime}}$. The individual $i$ is the dictator.

2. By assumption, $f_{\mathcal{D}}(\underline{\mathfrak{A}})$ is just the $A$-restriction of the reduced product of $\underline{\mathfrak{A}}$ with respect to $\mathcal{D}$. Since $\phi$ is free of negation, disjunction and universal quantification, an analysis of the proof of Łoś's theorem reveals that we must have

$$
\begin{aligned}
& f_{\mathcal{D}}(\underline{\mathfrak{A}}) \models \phi\left(a_{1}, \ldots, a_{m} ; a^{\prime}\right) \\
\Leftrightarrow \quad & \left\{i \in I: \mathfrak{A}_{i} \models \phi\left(a_{1}, \ldots, a_{m} ; a^{\prime}\right)\right\} \in \mathcal{D}
\end{aligned}
$$

for all $a_{1}, \ldots, a_{m}, a^{\prime} \in A$. Hence, as before one can show that the filter $\mathcal{D}$ contains a finite subset of $I$, viz. $C_{\vec{a}, a^{\prime}}$. But then, $\mathcal{D}=\left\{C \subseteq I: C^{\prime} \subset C\right\}$ for some $C^{\prime} \subseteq C_{\vec{a}, a^{\prime}}$. This $C^{\prime}$, necessarily a finite set, is the set of oligarchs.

\footnotetext{
${ }^{0}$ Corrected after publication.
} 
Already Lauwers and Van Liedekerke [17, p. 230, Property 4 (of aggregation functions)] had obtained a dictatorial impossibility theorem for preference aggregators that preserve certain non-universal formulae. However, their theorem is based on a syntactic condition which is quite restrictive as it entails that $A$ is countable and that $I$ is the set of nonnegative integers $\mathbf{N}$. (Lauwers' and Van Liedekerke's [17] proof strategy consisted essentially in constructing an aggregator based on a free ultrafilter which does not preserve the truth value of the non-universal formula in question, because the element which satisfies it does, by construction, not belong to $A$.) Our condition allows uncountable sets of alternatives and uncountable populations.

Moreover, even in the special setting of countably many alternatives and individuals, our condition is at least as general as the one of Lauwers and Van Liedekerke [17]:

Theorem 4.2. Let $I=\mathbf{N}$ and $A=\left\{\alpha_{i}\right\}_{i \in \mathbf{N}}$. For all $n \in \mathbf{N}$, let $\psi_{n}$ be the formula

$$
\left.\left.\left.\left.\ddot{(} \dot{\forall} \dot{x}_{1}\right) \ldots \dot{(\forall} \dot{x}_{m}\right) \ddot{(} \dot{\forall} \dot{y}\right) \dot{(} \phi\left(\dot{x}_{1}, \ldots, \dot{x}_{m} ; \dot{\alpha}_{n+1}\right) \dot{\wedge} \dot{(} \phi\left(\dot{x}_{1}, \ldots, \dot{x}_{m} ; \dot{y}\right) \dot{\rightarrow} \dot{\bigwedge}_{j=0}^{n} \dot{y} \dot{\neq} \alpha_{j}\right) \ddot{)} .
$$

If $T \cup\left\{\psi_{n}\right\}$ is consistent for all $n \in \mathbf{N}$, then there exists some $\underline{A} \in \Omega^{I}$ with finite witness multiplicity with respect to $\psi$.

Proof of Theorem 4.2. Suppose that $T \cup\left\{\psi_{n}\right\}$ is consistent for all $n \in \mathbf{N}$. Then there exists for every $n \in \mathbf{N}$ some model $\mathfrak{A}_{n}$ of $T \cup\left\{\psi_{n}\right\}$ with domain $A{ }^{16}$ Then, for every $k \in \mathbf{N}$ and arbitrary $a_{1}, \ldots, a_{m} \in A$, the set

$$
\left\{n \in \mathbf{N}: \mathfrak{A}_{n} \models \phi\left(a_{1}, \ldots, a_{m} ; \alpha_{k}\right)\right\}
$$

must contain $k-1$, but none of the integers $\geq k$. It is therefore finite. Since $A=\left\{\alpha_{k}\right\}_{k \in \mathbf{N}}$, we conclude that for all $a \in A$ and all $a_{1}, \ldots, a_{m} \in A$, the set

$$
\left\{n \in \mathbf{N}: \mathfrak{A}_{n} \models \phi\left(a_{1}, \ldots, a_{m} ; a\right)\right\}
$$

is finite. On the other hand, $\psi_{n}$ implies $\psi$, so each of the $\mathfrak{A}_{n}$ is a model of $\psi$. This proves that $\left\langle\mathfrak{A}_{n}\right\rangle_{n \in I}$ has finite witness multiplicity with respect to $\psi$.

It is not clear whether the converse of the Theorem holds. Is it absurd that $\left.\left.\left.T \vdash \dot{(\forall} \dot{x}_{1} \dot{)} \ldots \dot{(\forall} \dot{x}_{m}\right) \dot{(} \dot{\forall} \dot{z}\right) \dot{(} \phi\left(\dot{x}_{1}, \ldots, \dot{x}_{m} ; \dot{z}\right) \dot{\rightarrow} \dot{\bigwedge}_{j=1}^{m} \dot{z} \dot{\neq} \dot{x}_{j}\right)$, so that $T \cup\left\{\psi_{n}\right\}$ will be inconsistent (put e.g. $x_{1}=z=\alpha_{n+1}$ ) for every $n \in \mathbf{N}$, while there still exists some profile with finite witness multiplicity with respect to $\psi$ ? If so, then for the special case $I=\mathbf{N}$, the existence of a profile with finite witness multiplicity would be equivalent to the above straightforward generalisation of Lauwers' and Van Liedekerke's condition. Hence, proving or disproving the converse of the theorem is a worthwhile task for future research.

\footnotetext{
${ }^{16}$ For, by completeness, there exists for every $n \in \mathbf{N}$ some model $\mathfrak{A}_{n}$ of $T \cup\left\{\psi_{n}\right\}$ with domain $A_{n}$, relational interpretations $P^{m} \subseteq A_{n}^{\delta(m)}(m \in \mathbf{N})$ and pairwise distinct constant interpretations $c_{a}^{n} \in A_{n}(a \in A)$. Since $T \cup\left\{\psi_{n}\right\}$ is universal, the restriction of this relational structure to $\left\{c_{n}^{a}: a \in A\right\}$ will still be a model of $T \cup\left\{\psi_{n}\right\}$. Without loss of generality, one may assume that $c_{n}^{a}=a$ for all $a \in A$.
} 


\section{Discussion and applications}

As shown in a companion paper [12, in a model-theoretic framework for the analysis of aggregation problems the ultraproduct construction allows to improve our understanding of the correspondence between abstract aggregation rules in an Arrovian spirit and ultrafilters of decisive coalitions on the set of individuals. Whilst this construction immediately reveals why dictatorship results do not carry over to the infinite case - where free ultrafilters exist -, it opens up another source of impossibility results, which we have analyzed in this paper: Non-universal statements are generically not preserved under aggregation. This problem is, of course, hardly surprising from the vantage point of model theory (given that an important use of ultraproducts is the enlargement of a given structure). However, it challenges one of the usual conditions on aggregation rules - viz. that the aggregate model has exactly the same domain as the individual models (the factor domains of the ultraproduct) - , as this requirement can only be met for sufficiently rich theories if the ultrafilter of decisive coalitions is principal, i.e. the aggregation rule is dictatorial.

Let us finally consider some applications of our impossibility theorem (Theorem 4.1):

In preference aggregation, as already remarked by Lauwers and Van Liedekerke [17, p. 231], any Arrovian aggregator which preserves either continuity or the existence of upper bounds or lower bounds must be dictatorial. The reason is that one can devise profiles with finite witness multiplicity with respect to the formula expressing continuity of preferences, and there exist also profiles with finite witness multiplicity with respect to the formula describing the existence of an upper/lower bound. In particular, this yields an alternative proof of Campbell's theorem [3] (which asserts the impossibility of non-dictatorial, Arrovian and continuity-preserving aggregators - regardless of the electorate's cardinality).

As an application to propositional judgment aggregation à la Dietrich and List 4, consider a propositional judgment aggregator which satisfies Arrovian axioms. Suppose that the agenda is infinite, satisfies usual agenda richness conditions, and that the judgment aggregator preserves the existence of an implication-maximal (implication-minimal, resp.) proposition, so that if there exists for every individual some proposition in his/her judgment set which entails (is entailed by) all other propositions that he/she accepts, then the collective judgment set will also contain a proposition which entails (is entailed by) all other propositions in the judgment set. Then, the judgment aggregator must be oligarchic. Under stronger agenda conditions, the aggregator must even be dictatorial.

For an application to modal judgment aggregation, consider now a modal judgment aggregator satisfying Arrovian axioms. Suppose that there are infinitely many possible worlds and that the judgment aggregator preserves the existence of a universally accessible possible world, so that if for each individual there exists some possible world which is accessible from all other possible worlds, then there is also a possible world which is socially accessible from all other possible worlds. Then, the judgment aggregator must be oligarchic. Under stronger agenda conditions, the aggregator must even be dictatorial.

Another application lies in proofs of impossibility theorems for Arrovian aggregation of variational preferences (convex risk measures), cf. Herzberg [11]. 


\section{References}

[1] K.J. Arrow. Social choice and individual values, volume 12 of Cowles Commission Monographs. Wiley, New York, 2 edition, 1963.

[2] J.L. Bell and A.B. Slomson. Models and ultraproducts. An introduction. North-Holland, Amsterdam, 1969.

[3] D.E. Campbell. Intergenerational social choice without the Pareto principle. Journal of Economic Theory, 50(2):414-423, 1990.

[4] F. Dietrich and C. List. Arrow's theorem in judgment aggregation. Social Choice and Welfare, 29(1):19-33, 2007.

[5] F. Dietrich and Ph. Mongin. The premiss-based approach to logical aggregation. Les Cahiers de Recherche 886, HEC Paris, December 2007.

[6] F. Dietrich and Ph. Mongin. The premiss-based approach to judgment aggregation. Journal of Economic Theory, 145(2):562-582, 2010.

[7] P.C. Fishburn. Arrow's impossibility theorem: concise proof and infinite voters. Journal of Economic Theory, 2(1):103-106, 1970.

[8] U. Grandi and U. Endriss. First-order logic formalisation of Arrow's theorem. In Proceedings of the 2nd International Workshop on Logic, Rationality and Interaction (LORI-2009), volume 5834 of Lecture Notes in Computer Science, pages 133-146. Springer, Berlin, 2009.

[9] J.D. Halpern and A. Levy. The Boolean prime ideal theorem does not imply the axiom of choice. In D. Scott, editor, Axiomatic Set Theory, volume XIII, part 1 of Proceedings of Symposia in Pure Mathematics, pages 83-134. American Mathematical Society, Providence, RI, 1971.

[10] B. Hansson. The existence of group preference functions. Public Choice, 38(1):98-98, 1976.

[11] F.S. Herzberg. Social choice of convex risk measures through Arrovian aggregation of variational preferences. IMW Working Paper 432, Institute of Mathematical Economics, Bielefeld University, May 2010.

[12] F.S. Herzberg and D. Eckert. The model-theoretic approach to aggregation: Impossibility results for finite and infinite electorates. Mathematical Social Sciences, 64(1):41-47, 2012. doi:10.1016/j.mathsocsci.2011.08.004.

[13] F.S. Herzberg, L. Lauwers, L. Van Liedekerke, and E.S. Fianu. Addendum to L. Lauwers and L. Van Liedekerke, "Ultraproducts and aggregation". Journal of Mathematical Economics, 46(2):277-278, 2010.

[14] W. Hodges. Building models by games, volume 2 of London Mathematical Society Student Texts. Cambridge University Press, Cambridge, UK, 1985.

[15] A.P. Kirman and D. Sondermann. Arrow's theorem, many agents, and invisible dictators. Journal of Economic Theory, 5(2):267-277, 1972. 
[16] C. Klamler and D. Eckert. A simple ultrafilter proof for an impossibility theorem in judgment aggregation. Economics Bulletin, 29(1):320-328, 2009 .

[17] L. Lauwers and L. Van Liedekerke. Ultraproducts and aggregation. Journal of Mathematical Economics, 24(3):217-237, 1995.

[18] C. List and C. Puppe. Judgment aggregation: A survey. In P. Anand, P.K. Pattanaik, and C. Puppe, editors, The Handbook of Rational and Social Choice: An Overview of New Foundations and Applications, pages 457482. Oxford University Press, Oxford, 2009.

[19] A. Rubinstein. The single profile analogues to multi profile theorems: Mathematical logic's approach. International Economic Review, 25(3):719730, 1984.

[20] A. Rubinstein and P. Fishburn. Algebraic aggregation theory. Journal of Economic Theory, 38(1):63-77, 1986.

[21] P. Suppes. The pre-history of Kenneth Arrow's Social Choice and Individual Values. Social Choice and Welfare, 25(2-3):319-326, 2005.

[22] R. Wilson. On the theory of aggregation. Journal of Economic Theory, 10(1):89-99, 1975 . 